\title{
Computación ubicua al servicio de la educación
}

\author{
Edgar Rivadeneira Ramos \\ eribaden@ueb.edu.ec \\ Universidad Estatal de Bolívar \\ Guaranda - Ecuador
}

\section{RESUMEN}

Este trabajo muestra al lector una aproximación de la relación entre la computación ubicua y la educación, misma que define al modelo de educación ubicua, un paradigma académico que aprovecha la omnipresencia de la tecnología puesta al servicio de las personas.

El horizonte que de una u otra manera se plantea, muestra una problemática que pudiera ser considerada para un proceso de investigación que profundice en las capacidades de los sistemas, entre ellos informáticos, computacionales, académicos, cognitivos y dentro de estos últimos, la integración de la inteligencia artificial, la minería de datos académicos, todo en comunión con sistemas de gestión del aprendizaje.

Metodológicamente, este documento se basa en la revisión de un amplio compendio de documentos que luego de una valoración cualitativa, han sido considerados como relevantes, se llega al planteamiento de una discusión que deberá desembocar en una o varias líneas de investigación al respecto. Al final se plantean conclusiones, que desde la perspectiva del autor son prudentes de considerar.

Palabras claves: educación virtual; educación ubicua; computación ubicua; LMS; modelo de aprendizaje 


\title{
Ubiquitous computing at the service of education
}

\begin{abstract}
This paper shows the reader an approach to the relationship between ubiquitous computing and education, which defines the ubiquitous education model, an academic paradigm that takes advantage of the omnipresence of technology at the service of people. The horizon that in one way or another, shows a problem that could be considered for a research process that deepens the capabilities of systems, including computer, computational, academic, cognitive and within the latter, the integration of artificial intelligence, academic data mining, all in conjunction with learning management systems. Methodologically, this document is based on the review of a wide range of documents that, after a qualitative assessment, have been considered as relevant, leading to a discussion that should lead to one or more lines of research in this regard. At the end, conclusions are raised, which from the author's perspective are prudent to consider.
\end{abstract}

Keywords: virtual education; ubiquitous education; ubiquitous computing; LMS; learning model

Artículo recibido: 05 octubre. 2021 Aceptado para publicación: 02 noviembre 2021

Correspondencia: eribaden@ueb.edu.ec Conflictos de Interés: Ninguna que declarar 


\section{INTRODUCCIÓN}

Se parte de una revisión sistemática de literatura para conseguir un documento que se enfoca en el ambiente de la computación ubicua y su contribución y/o efecto en la educación, que de una u otra manera se ha convertido en los entornos de educación ubicua.

\section{Problema}

La introducción de la computación ubicua en la educación ha venido siendo un interés de muchos investigadores en las áreas de la educación y la tecnología; sin embargo, los resultados que se han podido revisar apuntan a la implementación de la U-Learning en un área o materia específica y puntual dentro de la formación de los estudiantes, tal vez porque no se ha dado aún un perfil holístico a la formación profesional bajo este concepto. Se considera que la educación a través de dispositivos móviles aporta sobre manera a la educación ubicua, de acuerdo a lo que menciona Gladys Lagos (2018, p 115):

"El aprendizaje móvil presenta beneficios como la accesibilidad, conectividad la ubicuidad es decir el acceso en cualquier momento y lugar, con lo que se rompen las barreras de tiempo y espacio. El m-learning constituye la versión mejorada del elearning (aprendizaje en línea o virtual), misma que esta universalizada en todos los campos de acción del ser humano."

En la investigación realizada por Lagos (2018), se establece que, en el Ecuador, y de forma particular en la Universidad de Guayaquil, Facultad de Filosofía, el 96,2\% de los encuestados hacen uso de dispositivos móviles en sus horas de clase, el $83 \%$ utiliza esta tecnología para buscar contenido referente a las materias que llevan; y, 89\% sostiene que el aprendizaje móvil debería ser aplicado en todas las asignaturas.

Rivero y Suárez (2017, p. 38), en su publicación refieren que “...existen algunos estudios, como el de Hwang, Wu, Zhuang \& Huang (2013), que afirman que se puede hablar de mejora en el rendimiento de los alumnos que aprenden con el enfoque del aprendizaje móvil (tabletas y dispositivos móviles) frente a aquellos que aprenden con el enfoque tradicional".

Durante los últimos tres periodos académicos, el autor de esta investigación, aplicó una encuesta a una muestra (estratificada para 12 carreras) de 329 estudiantes de la Facultad de Ciencias Administrativas, Gestión Empresarial e Informática de la Universidad Estatal 
de Bolívar, con el propósito de conocer la opinión de los alumnos sobre el proceso de educación virtual a la que se han visto sometidos a raíz del aparecimiento de la pandemia producida por el COVID-19.

Entre los resultados más importantes o interesantes de analizar se consideran a la área de residencia de los estudiantes, la población estudiantil se divide casi equitativamente entre el sector rural con un $49,8 \%$ y en el sector urbano con un 50,2\%; el acceso a la internet se da a través de la contratación de un servicio fijo $86,9 \%$ y con la compra de los llamados megas (termino vulgar para referirse a la cantidad de datos, medidos en megabytes, en una conexión celular) en un $13,1 \%$; además, de que quienes tienen acceso por medio de un servicio fijo, el 77,2\% lo tienen en sus hogares; los restantes lo hacen a través del uso en casas de algún familiar, centros comunitarios o locales de alquiler. La asistencia a las actividades síncronas que se desarrollan es a través de dispositivos móviles (tablets y smartphones) con un 29,2\% y con computadores portátiles (laptops) en un 55,3\%. Se puede entender que la potencialidad de los estudiantes para sumergirse en una educación virtual es alta y que es posible pensar en un entorno ubicuo para su formación. Por último, se ha tratado de identificar los aspectos más relevantes por el nivel de dificultad o problemas que han debido superar los estudiantes para desenvolver sus actividades virtuales, mismas que se pueden ver resumidas en el siguiente gráfico.

\section{Gráfico $\mathbf{N}^{\circ} 1$}

\section{Dificultades para desarrollar las actividades virtuales}

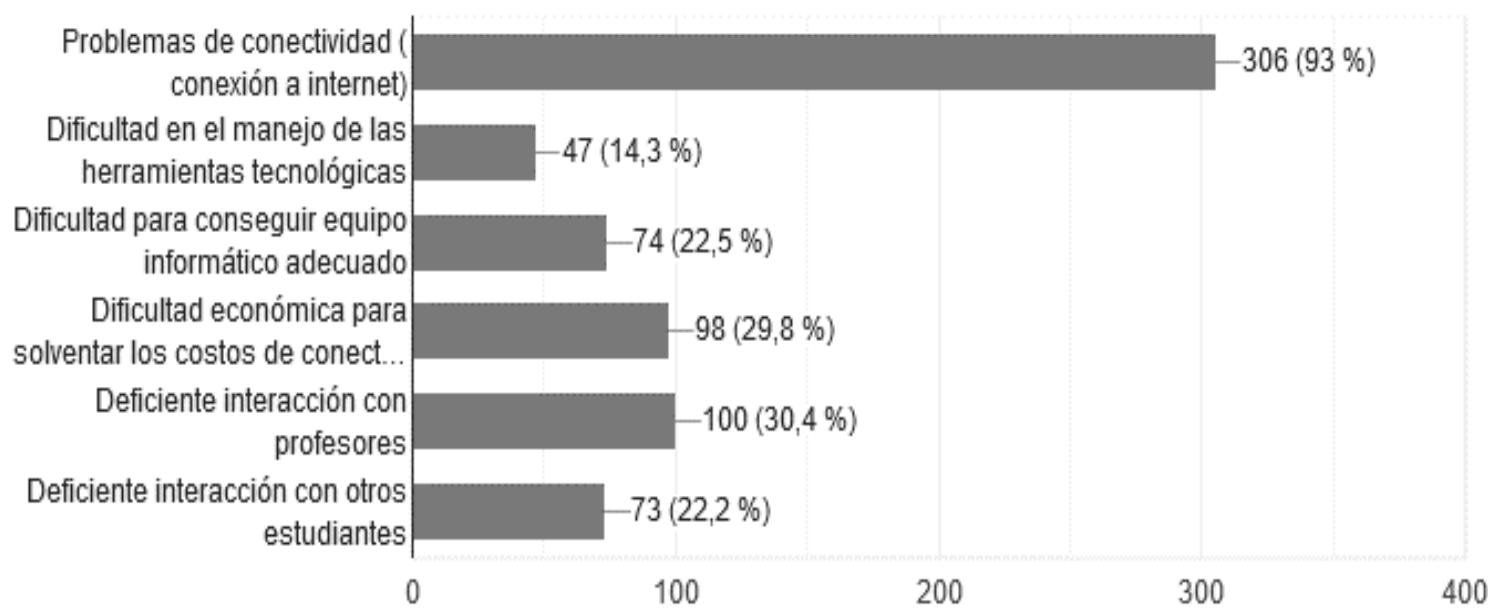

Fuente: encuesta aplicada a 329 estudiantes de la Fac. Ciencias Administrativas, Gestión Empresarial e Informática, UEB, ponderación a mayo de 2021

Autor: Edgar Rivadeneira Ramos 
Se puede ya notar la relevancia de los problemas: la conectividad, la deficiente interacción con los profesores y las dificultades económicas, resultan ser los más destacados. De éstos, el que más le interesa al autor es el que hace referencia a la relación con los profesores, cuando el 30,4\% de los alumnos señala la existencia de una deficiente interacción; por otro lado, es llamativa la percepción estudiantil, 51,4\%, de que los docentes están preparados didácticamente para afrontar un proceso académico virtual, y $57,5 \%$ de que están bastante bien preparados para usar la tecnología asociada a esta modalidad educativa.

Con la información recolectada, se pueden ir definiendo oportunidades y problemáticas; oportunidades desde la capacidad de los estudiantes de acceder a la internet con dispositivos móviles y computadores portátiles, que ayudan a que sean los dueños del tiempo y el espacio para su profesionalización; otra oportunidad, la capacidad de los profesores en el uso de la tecnología y didáctica en la educación virtual (desde la perspectiva de los estudiantes, quienes son los beneficiarios directos); y como problema, la deficiente interacción con los profesores, aspecto que eventualmente podría ser superado con una orientación curricular apoyada en la computación ubicua como modalidad de estudio (educación ubicua), aspecto que va más allá del modelo educativo que mantenga la institución.

\section{Planteamiento del problema}

Antes de definir un problema en específico para este trabajo, es conveniente conocer lo que otros autores proponen como perfiles de investigaciones futuras.

En este este contexto, Gabriel Ramírez Villegas (2019), en su tesis doctoral propone En cuanto al U-Learning los diferentes autores referenciados y consultados sugieren la necesidad de desarrollar nuevas formas de implementar el U-Learning como una nueva forma y estrategia de desarrollar los procesos de aprendizaje, llevando más allá la educación, para romper las barreras del tiempo, el espacio, los contextos y los medios, llevando el aprendizaje a no tener costuras, ni limites, que el aprendizaje se realice para toda la vida y se aplique en cualquier momento y contexto. Los autores de U-Learning plantean la necesidad de diseñar y desarrollar nuevos modelos, metodologías, estrategias, pedagogías que incluyan el U-Learning en los procesos de aprendizaje como una forma de mejorar, revolucionar y evolucionar el aprendizaje, y por ende la educación de forma prospectiva, con una visión futura, es decir preparar a las personas actuales para un nuevo 
mundo impregnado por la tecnología en todos los aspectos cotidianos de la sociedad. ( $\mathrm{p}$ $164,165)$.

Gustavo Moreno (2020) plantea la posibilidad de trabajos futuros en referencia a “Aspectos tecnológicos en la solución de u-learning. Involucrar la aplicación de inteligencia artificial a los videos (por ejemplo, para búsquedas inteligentes de información dentro del video, analítica, etc) y a la solución. Ampliar la aplicación sensible al contexto".

Byron Hidalgo Cajo (2018), en uno de sus trabajos concluye que "La educación ha cambiado su enfoque centrándose en los estudiantes de lo que deben aprender y cómo hacerlo, la minería de datos es la solución adecuada para cubrir estas necesidades, teniendo en cuenta que el análisis de datos permite conocer las fortalezas y debilidades de los estudiantes, este conocimiento permite crear métodos educativos adaptativos establecidos en la mejora del proceso enseñanza aprendizaje”.

Sobre el análisis de las sugerencias y conclusiones de los autores mencionados, se presenta de forma arriesgada el planteamiento de un potencial problema como la dificultad que tienen los estudiantes del nivel superior o universitario, para desarrollar las actividades académicas en un entorno virtual, al no contar con un modelo tecnológicoeducativo que les procure facilidades para la realización de sus actividades atendiendo sus reales necesidades, además de considerar su libertad de elección de tiempo y espacio.

\section{ESTADO DEL ARTE}

Desde que Mark Weiser concibiera la idea y definición de la "Computación Ubicua", la posibilidad de integrar de forma omnipresente a la tecnología en las actividades humanas, haciéndola transparente para todos, la idea de combinar la ubicuidad en la educación con el uso de la computación ubicua ha dado lugar a la educación ubicua, adaptando la terminología en "Aprendizaje Ubicuo" o U-Learning.

\section{Apreciaciones y conceptos}

Según el Dr. Santiago Acosta, Rector de la Universidad Técnica Particular de Loja (UTPL) en el Ecuador, la evolución de la educación a distancia en el país, debe atender aspectos como el contacto indicando que "la principal transformación es la virtualización con plataformas que permiten desarrollar clases síncronas", la inclusión educativa, la tendencia digital "La tendencia dominante es la educación en entornos virtuales, el uso de recursos tecnológicos y enfoques pedagógicos para trasladar la educación presencial a 
una educación en línea" y la prioridad al estudiante, centrándose en un "modelo educativo es dar prioridad al estudiante y atender a sus necesidades" (UTPL, 2020).

De la mano con lo expuesto, está la transición necesaria para integrarse a esta nueva modalidad educativa, al respecto, el MSc. Xavier Mosquera R., Decano de estudios en modalidad presencial de la Universidad Tecnológica Empresarial de Guayaquil, indica que

“...nos encontramos con un escenario en el cual el acceso a los recursos tecnológicos que son necesarios para la educación en línea, se convierte en un factor de alto impacto que amplía la brecha, ya no digital, sino educativa y social, que impide que las facilidades que brinda este nuevo paradigma en la educación, sea de acceso limitado.” (Mosquera R., 2020).

La necesidad de los individuos de formarse, de conseguir un título profesional que de una u otra manera le permita tener mayores posibilidades de crecimiento económico, social o del tipo que fuere, debe ser entendido como la posibilidad de crear nuevas oportunidades de acción de las IES, en donde el control de las actividades sea compartido entre todos los actores involucrados, instituciones e individuos, con las responsabilidades clarificadas y asociadas a cada uno.

En un estudio reciente sobre la percepción de la educación virtual en el nivel superior, desde la mirada de los estudiantes de la Universidad Estatal de Bolívar, se menciona que "El contexto actual para las Instituciones de Educación Superior (IES), ha originado la necesidad institucional de adaptarse a un ambiente de aprendizaje virtual, recurriendo a un proceso de migración del medio tradicional a uno basado en entornos virtuales, proceso que conlleva a requerir de recursos, planificación y capacitación de los involucrados, de manera especial de docentes y estudiantes. Por tanto, las IES deben estar preparadas para este nuevo reto garantizando una educación de calidad." (Chavez et al. 2021, p 16)

El no prepararse para enfrentar un nuevo modelo educativo, incluso sin la presencia de factores sanitarios en el entorno (aunque ahora lo ha acelerado), sino simplemente considerando el avance tecnológico, pedagógico y de formación en el mundo, obliga que 
tanto las IES como los potenciales beneficiarios, se preparen a afrontar noveles maneras de interactuar.

Las dificultades de asumir un modelo de educación que rompe el estereotipo de la presencialidad, sustituyéndolo con una estructura intangible, a veces complicada de entender, difícil de asumir desde la perspectiva de los profesores (sobre todo los de edad avanzada), entre otros factores o características, definen una problemática actual que debe ser superada para lograr un compromiso relacional entre la tecnología y la academia en consideración de la nueva "realidad", que beneficie a profesores y estudiantes de las IES, y por consiguiente al desarrollo de la sociedad.

Cuando Robertson y Good (2005, como se citó en Stanojević, Cenić, y Cenić 2018) manifiestan que "el uso de las computadoras abre nuevas oportunidades de aprendizaje activo en las que el estudiante como investigador asume la responsabilidad de su propio aprendizaje. Pero el maestro sigue siendo el titular creativo de la enseñanza, y el uso de nuevas tecnologías le brinda nuevas oportunidades para fomentar la expresión creativa en los niños. Asimismo, es importante destacar el impacto positivo en la motivación para el trabajo y el aprendizaje que pueden tener las nuevas tecnologías, y como lo confirman numerosos estudios", están dando una pauta de las posibilidades de la integración de la “computación” en la educación, haciendo hincapié en el papel que los involucrados juegan el contexto educativo.

Por otro lado, la existencia de conceptos como el de aprendizaje adaptivo, que "se basa en el supuesto de que el sistema inteligente creado podrá adaptarse al proceso de aprendizaje del estudiante, lo que resultará en una experiencia de aprendizaje mejor y más eficaz" (Costa et al. 2020), y de la educación virtual, cuando (Crisol-Moya, HerreraNieves, and Montes-Soldado 2020) cita la definición de Morales, Fernández, \& Pulido (2016) al respecto, "La educación virtual es una estrategia de alto impacto en la mejora de la cobertura, pertinencia y calidad educativa en todos los niveles y tipos de formación, debido a sus características multimediales, hipertextuales e interactivas"; (Báez and Beaufond 2019) definen a la educación ubicua (U-Learning) como la que "permite el aprendizaje en cualquier momento y en cualquier lugar, dependiendo del entorno de aplicación y apoyado por herramientas digitales que permiten la inclusión de diferentes actores dentro del proceso de formación”, gráficamente se puede ver en la figura número 1. Algo también interesante es la rudimento de (Costa et al. 2020) al señalar que "la 
educación a distancia surge como un nuevo modelo educativo, con el fin de apoyar la propagación del conocimiento de una manera más ágil, facilitando el acceso a los aprendizajes"

\section{Principales investigaciones previas}

Existen varias investigaciones que muestran la posibilidad de incluir la computación ubicua a través de un modelo de educación ubicua, Mauricio Ramírez Villegas "Modelo U-Learning Soportado por las Experiencias de Aprendizaje y el Aprendizaje Conectivo para la Educación Superior Virtual - U-CLX"; Gustavo Moreno López "Modelo de Ulearning basado en plataformas de TV everywhere").

Otros trabajos interesantes son los de Joung-Souk Sung "U-Learning Model Design Based on Ubiquitous Environment”, y el de Mayela Coto, César A. Collazos y Sonia Mora Rivera "Collaborative and Ubiquitous Model to Support Teaching and Learning Processes in Iberoamerica".

Todos estos trabajos, entre otros, proponen formas de trabajo para un modelo de educación ubicua, de una u otra forma se está consolidando el marco genérico de este tipo de academia, una que priorice las inclinaciones, gustos, intereses, potencialidades y limitaciones de los clientes: los estudiantes.

\section{RESULTADOS Y DISCUSIÓN}

La computación ubicua, se presenta como una aplicación de las ciencias de la computación o la informática, en cualquier ambiente y con la posibilidad cierta de uso de todas sus herramientas disponibles, y al servicio de cualquier área del quehacer humano. La educación es el eje de todo proceso de desarrollo, sin educación no se podría llegar a generar nuevas y mejores formas de producción y satisfacción de necesidades individuales y/o colectivas; siendo así, no es de sorprender que la tecnología busque ser un puntal en el proceso académico.

Sin embargo, la aplicabilidad de un modelo educativo-tecnológico que permita un desenvolvimiento completo de los estudiantes, bajo un modelo integrador y holístico está aún en desarrollo; los autores citados han procurado sentar las bases metodológicas o teóricas de lo que deberías ser un entorno ubicuo en la educación; se han planteado un par de posibilidades que viabilizan un modelo funcional, que de una u otra manera consolidad la teoría en la práctica.

Se puede adelantar la idea de una solución que integre al modelo de educación ubicua, el 
componente de los sistemas cognitivos; esta posibilidad permitiría incluir en los LMS actuales un entorno de inteligencia artificial, la minería de datos educativos, el aprendizaje automático; estos elementos tendrían la responsabilidad de recolectar, medir, evaluar y aprender cuales son los comportamientos de los estudiantes, sus preferencias académicas, sus inclinaciones profesionales e incluso sus limitaciones de tiempo, entre otras variables, y con esa data se tendrá la capacidad de tomar decisiones que favorezcan el proceso formativo de los estudiantes al comprender su necesidades.

\section{CONCLUSIÓN O CONSIDERACIONES FINALES}

La tecnología y su desarrollo es, sin ser el único factor, fundamental para encaminar un crecimiento sostenido de cualquier actividad humana, y entre ellas educación. En ese contexto, la realidad de tener al alcance de todos, en todo momento y en cualquier lugar un sistema omnipresente de computación y sus herramientas, resulta determinante para que los individuos sean capaces de lograr hacer más y mejor las actividades que tengan a su cargo.

Los trabajos revisados coinciden en el hecho de que la computación ubicua, incluida en los procesos académicos, puede convertirse en una herramienta capaz de potencializar las formas de profesionalizar a los estudiantes, y sobre todo en el nivel de educación superior. Se concuerda en que aún fata mucho que investigar y mucho que definir y desarrollar; el futuro es ahora, ya se habla de la Z-Learning, un nuevo modelo, un nuevo paradigma de educación con un uso extensivo de la tecnología, un área que debe empezarse a explotar.

\section{LISTA DE REFERENCIAS}

Báez, Carmen, and Clifton Beaufond. 2019. “A Review of Ubiquitous Learning.” Revista Iberoamericana de Educación a Distancia 22:325-44.

Chávez, Margoth, Vladimir Rivera, and Giovanny Haro. 2021. "Percepción De La Educación Virtual En Instituciones De Educación Superior 2020 - 2020.” Revista de Investigación Enlace Universitario 20(1):8-21. doi: 10.33789/enlace.20.1.81.

Coto, Mayela, César A. Collazos, and Sonia Mora-Rivera. 2016. "Collaborative and Ubiquitous Model to Support Teaching and Learning Processes in Iberoamerica." Revista de Educación a Distancia (RED) (48). doi: 10.6018/red/48/10.

Costa, Roberto D., Gustavo F. Souza, Ricardo A. M. Valentim, and Thales B. Castro. 2020. "The Theory of Learning Styles Applied to Distance Learning." Cognitive Systems Research 64:134-45. doi: 10.1016/j.cogsys.2020.08.004. 
Crisol-Moya, Emilio, Liliana Herrera-Nieves, and Rosana Montes-Soldado. 2020. "Virtual Education for All: Systematic Review." Education in the Knowledge Society 21:1-13. doi: 10.14201/eks.20327.

Hidalgo Cajo, Byron G. 2018. "Data Mining in Learning Management Systems in University Education.” Campus Virtuales 7(2):115-28.

Lagos, Gladys Gioconda. 2018. “El M- Learning, Un Nuevo Escenario En La Educación Superior Del Ecuador." INNOVA Research Journal 3(10.1):114-22. doi: 10.33890/innova.v3.n10.1.2018.859.

Moreno, Gustavo. 2020. "Modelo de U - Learning Basado En Plataformas de TV Everywhere Modelo de U - Learning Basado En Plataformas de TV Everywhere.” Mosquera R., X. (Julio de 2020). Universidad Tecnológica Empresarial de Guayaquil. Obtenido de La brecha digital: su impacto en la educación en línea: https://www.uteg.edu.ec/la-brecha-digital-su-impacto-en-la-educacion-en-linea/

Ramírez, Mauricio. 2019. "Modelo U-Learning Soportado Por Las Experiencias de Aprendizaje y El Aprendizaje Conectivo Para La Educación Superior Virtual - UCLX Gabriel Mauricio Ramirez Villegas.”

Rivero, Carol, and Cristóbal Suárez. 2017. "Mobile Learning Y El Aprendizaje De Las Matemáticas: El Caso Del Proyecto MATI-TEC En El Perú.” Tendencias Pedagógicas 30:37-52.

Stanojević, Dragana, Dragan Cenić, and Stojan Cenić. 2018. “Application of Computers in Modernization of Teaching Science." International Journal of Cognitive Research in Science, Engineering and Education 6(2):89-104. doi: 10.5937/ijcrsee1802089S.

Sung, Joung-souk. 2009. "U-Learning Model Design Based on Ubiquitous Environment.” International Journal of Advanced Science and Technology 13:7788.

UTPL. (Octubre de 2020). 4 claves para entender la evolución de la Educación a Distancia en el país. Obtenido de https://noticias.utpl.edu.ec/4-claves-para-entender-laevolucion-de-la-educacion-a-distancia-en-el-pais

Weiser, Mark. 1999. “The Computer for 21st Century.” Pervasive Computing 1:19-25. 